\title{
SISTEMA AUTOMÁTICO PARA A MEDIÇÃO DA CONCENTRAÇÃO DOS NÚCLEOS DE CONDENSAÇÃO DE NUVENS POR VISÃO COMPUTACIONAL
}

\author{
Francisco Geraldo de Melo Pinheiro* \\ fgmpinheiro@hotmail.com
}

\author{
Paulo Cesar Cortez ${ }^{\dagger}$ \\ cortez@deti.ufc.br
}

\author{
João Cesar Moura Mota ${ }^{\dagger}$ \\ mota@gtel.ufc.br \\ *Curso de Física \\ Universidade Estadual do Ceará \\ Fortaleza, Ceará, Brasil \\ ${ }^{\dagger}$ Departamento de Teleinformática \\ Universidade Federal do Ceará \\ Fortaleza, Ceará, Brasil
}

\section{ABSTRACT}

Automatic System for Measuring the Concentration of Clouds Condensation Nuclei by Computer Vision

In this paper is described an automatic system for measuring the concentration of cloud condensation nuclei by computer vision, the procedures and infrastructure of the laboratory for evaluation and, finally, it is compared its measurements with other equipment taken as a reference. The measurement system developed uses for such, digital image processing techniques. The process consists of capturing a sample of atmospheric air inside a static thermal diffusion chamber supersaturated with water vapor. In this condition, the molecules of water vapor condense on the aerosol captured in the air, producing water droplets. These droplets fall by gravity and cross a laser light beam, which defines a sampling volume, making them visible. Series of images of this process is digitized and processed for identification and isolation of the droplets present in the sample volume. Then, these droplets are automatically counted and their concentration is calcu-

Artigo submetido em 12/07/2010 (Id.: 01169)

Revisado em 25/11/2010, 13/04/2011

Aceito sob recomendação do Editor Associado Prof. Sebastian Yuri Cavalcanti Catunda lated. The threshold, distance and watershed transformations techniques are used. An efficient methodology for determination of sample volume is also presented. The results of experiments performed under controlled conditions indicate that the followed procedures are appropriate in determining the concentration of cloud condensation nuclei and that the equipment developed is effective in high concentrations where other equivalent instruments are no longer reliable due to the effect of overlap of droplets in the images analyzed.

KEYWORDS: digital image processing; watershed transformation; distance transform; atmospheric aerosol; meteorological instrumentation; cloud condensation nuclei.

\section{RESUMO}

Neste artigo é descrito um sistema para medição automática da concentração de núcleos de condensação de nuvens por visão computacional, os procedimentos e a infraestrutura de laboratório para sua avaliação e, por fim, compara suas medições com as de outro equipamento considerado como referência. O sistema de medição desenvolvido utiliza para tal, técnicas de processamento digital de imagens. O processo consiste na captura de uma amostra do ar atmosférico dentro 
de uma câmara de nuvens estática por difusão supersaturada de vapor de água. Nesta condição, as moléculas do vapor de água condensam sobre os núcleos de condensação de nuvens presentes no ar capturado, produzindo gotículas de água. Estas gotículas ao caírem por gravidade cruzam um feixe de luz LASER, que define um volume de amostragem, o que as tornam visíveis. Uma série de imagens deste processo é digitalizada e processada para identificação e isolamento das gotículas presentes no volume de amostragem. Em seguida, estas gotículas são automaticamente contadas e a sua concentração é calculada. As técnicas de binarização por limiar, transformada de distância e transformada watershed são utilizadas. Uma metodologia eficiente para determinação do volume de amostragem também é apresentada. Os resultados, dos experimentos realizados em condições controladas, indicam que os procedimentos aplicados são adequados na determinação da concentração dos núcleos de condensação de nuvens e que o equipamento desenvolvido é efetivo em altas concentrações em que outros equipamentos equivalentes já não são confiáveis devido ao efeito da sobreposição de gotículas nas imagens analisadas.

PALAVRAS-CHAVE: processamento de imagens digitais; transformada watershed; transformada distância; aerossol atmosférico; instrumentação meteorológica; núcleo de condensação de nuvens.

\section{INTRODUÇÃO}

Na atmosfera da Terra são encontradas partículas e componentes químicos de origem natural e antropogênica (Verdes, 2007). Esses elementos, suspensos na atmosfera, chamados de aerossóis atmosféricos, juntamente com a energia solar, a energia geotérmica e com as condições dos oceanos, criam um conjunto de fatores que modulam o clima da Terra (Vianello and Alves, 1991; Roberts et al., 2001; Rosenfeld, 2006).

Estudos recentes indicam que uma mudança climática em escala global está ocorrendo em um ritmo jamais observado (Loarie et al., 2009). Suas consequências já são percebidas em diversas partes do planeta. As razões para tal mudança são diagnosticadas e apresentadas no documento "Climate Change 2007: The Physical Science Basis". A forte presença de aerossóis de origem antropogênica na atmosfera da Terra é apontada como uma importante causa (Working Group I of the Intergovernmental Panel on Climate Change, 2007).

Existem diversas classes de aerossóis de acordo com sua origem, tamanho, e composição química. Dentre essas, aquela composta por aerossóis que servem como núcleos de condensação de nuvens (CCN - Cloud Condensation Nuclei) são de fundamental importância, pois definem as propriedades radiativas e a evolução das nuvens (Khain, 2009). Assim, conhe- cer a concentração dos $\mathrm{CCN}$ é fundamental para compreensão do comportamento das nuvens e, consequentemente do clima da Terra (McMurry, 2000). Uma forma de se medir a concentração deste tipo de partícula é torná-la opticamente detectável. Para tal, submete-se o aerossol a uma atmosfera supersaturada de vapor d'água, dentro de uma câmara de nuvens, e observa-se a formação de gotículas de água a partir da condensação do vapor na superfície do aerossol. A forma de se observar depende do tipo e tamanho das partículas que constituem o aerossol, podendo ser indireta, através da observação do espalhamento que as gotículas provocam em um feixe de luz, ou direta, através da análise de uma imagem fotográfica das gotículas formadas. Diversos contadores de CCN (CCNC) já foram desenvolvidos, podendo serem divididos entre os de fluxo contínuo e os estáticos, dependendo da cinética da parcela da atmosfera dentro do instrumento. Nenes et al estudaram teoricamente o comportamento de quatro tipos destes instrumentos (Nenes et al., 2001).

Nos primeiros CCNC a concentração de gotículas é obtida através da contagem visual direta usando técnicas fotográficas, mas posteriormente técnicas para medir o espalhamento de luz foram inseridas (Twomey, 1963) o que permitiu a automatização do processo. A técnica fotográfica de análise visual direta tem limitações claras, como a de se analisar milhares de fotos manualmente. As técnicas de espalhamento são baseadas na existência de uma relação entre a concentração de partículas e a intensidade da luz espalhada quando essas são iluminadas por um feixe de LASER. Essa relação, entretanto, é bastante complexa, tornando-se necessária a utilização de equações empíricas com coeficientes a serem determinados experimentalmente. Além disso, essa metodologia introduz incertezas intrínsecas associadas ao fato de que o espalhamento de luz depende não somente da concentração das partículas, mas também das "seções de choque"e, portanto, da geometria de cada partícula no caminho do feixe (Oliveira and Vali, 1995). Outra questão importante é o ruído intrínseco ao sistema eletrônico de fotodetecção que deteriora a relação sinal ruído, principalmente em situações de baixa concentração (Pinheiro, 1999).

Os equipamentos mais modernos incorporam análise fotográfica associada a processamento digital de imagens. Entretanto, as técnicas utilizadas normalmente subestimam a concentração devido ao fenômeno de coincidência (Frank et al., 2006), sendo acuradas somente até concentrações de $600 / \mathrm{cm}^{3}$, que são muito baixas em comparação com as encontradas em muitas situações de interesse (Andreae et al., 2004).

No presente artigo propõe-se um procedimento de análise de imagens que envolve binarização, transformada de distância e transformada watershed na determinação do número de CCN em um CCNC de câmara de nuvens estática por difu- 
são térmica (CCNC-SDCC). Essa proposição é avaliada através da comparação do desempenho de um instrumento construído pelos autores com um outro de precisão conhecida, ambos atuando simultaneamente em condições controladas com diferentes tamanhos e concentrações de aerossóis.

\section{O CCNC DE CÂMARA DE NUVENS ES- TÁTICA POR DIFUSÃO}

O CCNC de câmara de nuvens estática por difusão (CCNCSDCC) é um instrumento que tem a finalidade de medir a concentração dos núcleos de condensação de nuvens em uma determinada supersaturação de vapor de água. O princípio de funcionamento desse equipamento consiste em criar uma região de pressão parcial de vapor $P_{v}$, maior do que a pressão de saturação de vapor d'agua $P_{s v}$, na região central da câmara de nuvens, ou seja, uma umidade relativa, $U R=\left(P_{v} / P_{s v}\right) \cdot 100 \%$, maior do que $100 \%$ nessa região. A pressão de saturação de vapor d'agua é a pressão em que o vapor se liquefaz em condições ideais, sendo uma propriedade que depende da temperatura de forma não linear (Wagner and PruSS, 2002). Nesta condição de supersaturação positiva, $S=U R-100 \%$, gotículas de nuvens se originam a partir da condensação do vapor de água na superfície dos CCN presentes na câmara, processo este chamado de nucleação heterogênea, quando se diz que o CCN está ativado. Um feixe de luz LASER atravessa o interior da câmara na região onde a supersaturação é máxima, criando um volume de amostragem cilíndrico, permitindo que imagens digitais das gotículas originadas da ativação dos $\mathrm{CCN}$ possam ser obtidas e analisadas.

O CCNC-SDCC é constituído de um cilindro de $10,0 \mathrm{~cm}$ de diâmetro e $1,0 \mathrm{~cm}$ de altura, de parede lateral feita de material isolante térmico e impermeável. O gradiente de temperatura, necessário para se obter uma determinada supersaturação, é obtido a partir do controle da diferença de temperatura entre placas de alumínio que fecham as extremidades inferior e superior da cavidade cilíndrica. A placa inferior é acoplada a dois resfriadores estáticos (pastilhas Peltier) e o gradiente desejado é mantido através de um controlador de temperatura do tipo proporcional, integral e diferencial (PID). Papéis absorventes, aderidos nas placas de alumínio, são abastecidos por um reservatório de água destilada, o que mantém uma umidade igualmente distribuída. $\mathrm{O}$ ar com os aerossóis que se deseja quantificar é admitido na câmara através do acionamento de uma bomba. Posteriormente a desativação da bomba a câmara é selada até que o sistema entre em equilíbrio.

O diagrama esquemático do CCNC-SDCC é apresentado na Figura 1. Na parte superior (a), destacam-se a vista superior da câmara de nuvens, uma fonte de luz LASER $(635 \mathrm{~nm})$, a câmera para o registro das imagens além da bomba, válvu- las e controlador que comanda a entrada de ar atmosférico para o interior da câmara de nuvens. Na parte inferior (b), destacam-se a vista lateral da câmara de nuvens, o posicionamento das pastilhas Peltier, o papel saturado com água, os sensores de temperatura, o controlador PID de temperatura e o reservatório de água.
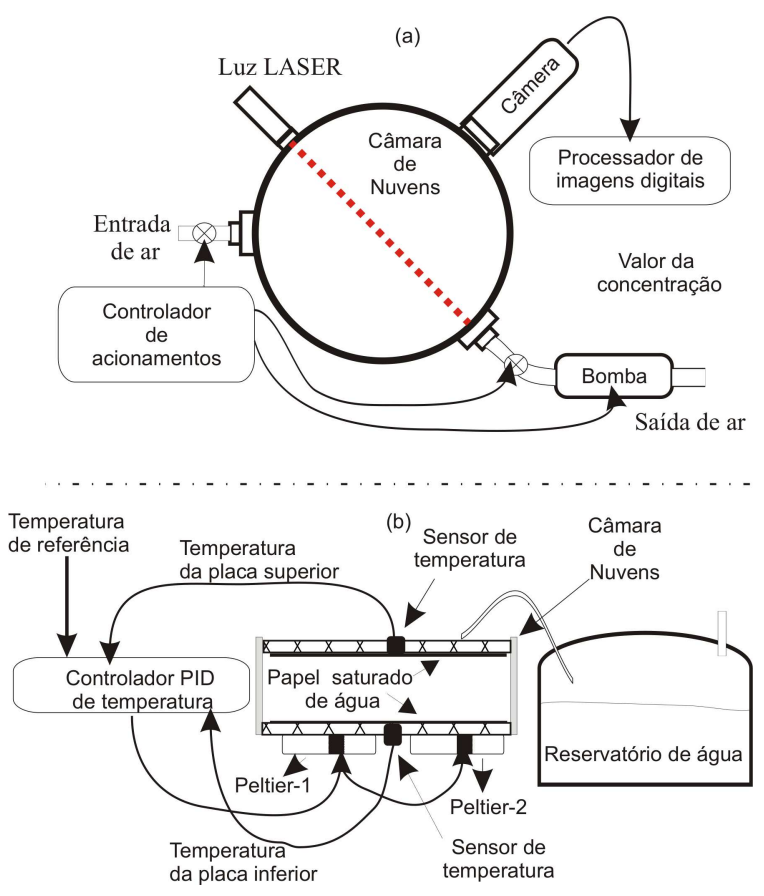

Figura 1: Diagrama esquemático do CCNC-SDCC, (a) vista superior da câmara de nuvens; (b) vista lateral.

A condição de supersaturação máxima, aproximadamente a meia altura da câmara, é obtida através da difusão de umidade a partir das duas placas mantidas em diferentes temperaturas. O processo de difusão garante um gradiente de pressão parcial de vapor e temperatura na câmara (Frank et al., 2006).

Os perfis estimados de (a) temperatura, (b) pressão parcial de vapor $\left(P_{v}\right)$ e de saturação de vapor $\left(P_{s v}\right)$ e (c) de supersaturação $(S)$ na câmara de nuvens estão mostrados na Figura 2. Estes perfis foram calculados considerando-se a temperatura da placa superior de $22,37^{\circ} \mathrm{C}$ e a temperatura da placa inferior de $17,51^{\circ} \mathrm{C}$. Os gradientes constantes de temperatura e pressão (Fig. 2 a e b) são presumidos a partir dos valores de temperatura medidos nas placas inferior e superior da câmara de nuvens e partem da suposição de que a pressão parcial de vapor nas regiões imediatamente próximas às placas é igual a pressão de saturação para as respectivas temperaturas. A pressão de saturação de vapor é obtida a partir dos valores na literatura (Wagner and PruSS, 2002; Frank et al., 2006) para as temperaturas correspondentes. Na Figura 2 (c) é 
apresentada a curva de supersaturação dentro da câmara de nuvens, derivada a partir de $P_{v}$ e $P_{s v}$, mostrando um perfil aproximadamente parabólico com o máximo a meia altura da câmara. O valor máximo de supersaturação é determinado, dessa forma, pelos valores das temperaturas das superfícies superior e inferior da câmara de nuvens.

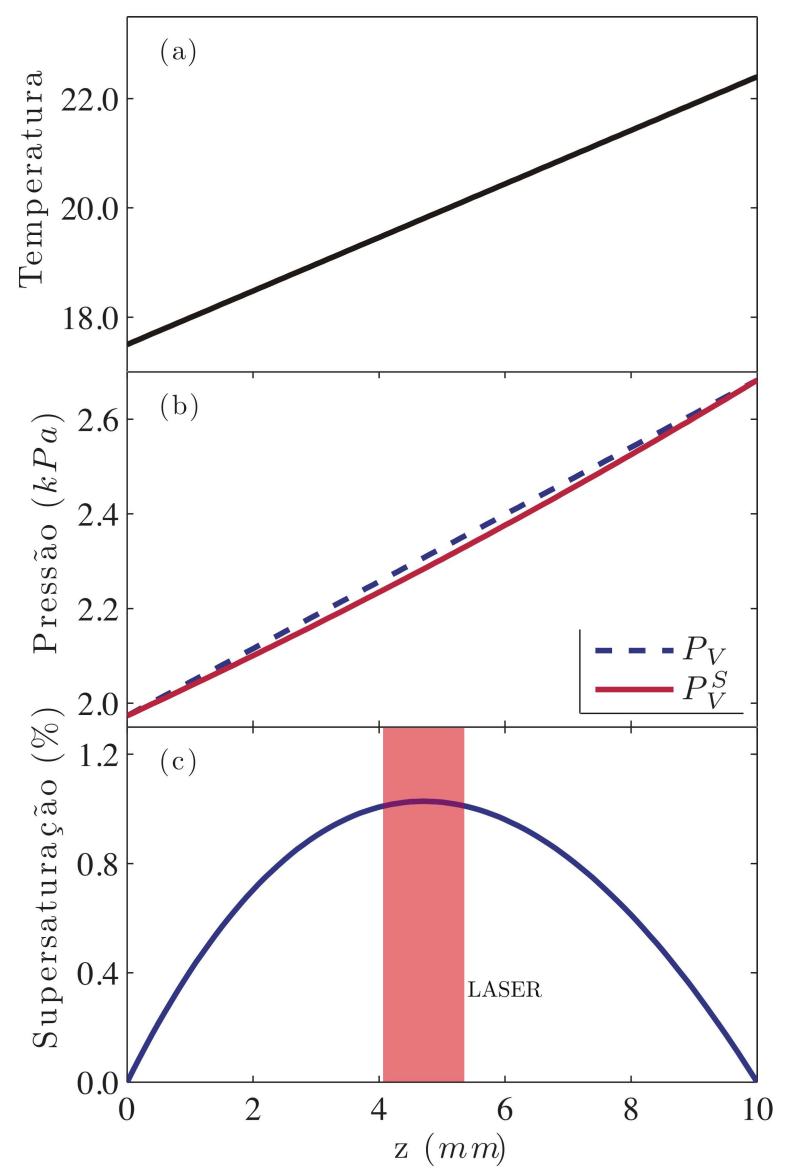

Figura 2: Perfil vertical de temperatura, (a) pressão; (b) de supersaturação; (c) dentro da câmara de nuvens.

A altura $z$ dentro da câmara de nuvens, onde a supersaturação é máxima, é a região de interesse para análise, pois, é nessa região onde o maior número de $\mathrm{CCN}$ presentes na câmara serão ativados. Um feixe cilíndrico de luz LASER então ilumina as gotículas formadas nessa região e a câmera registra a imagem para processamento.

O processo de ativação dos $\mathrm{CCN}$ é um processo dinâmico e o número de gotas na região de interesse cresce rapidamente, até que todos os CCN estejam ativados. Depois esse número cai gradativamente, devido à ação da gravidade. Várias fotografias são registradas durante esse processo, e o número de $\mathrm{CCN}, N_{g}$, é determinado utilizando a fotografia de maior contagem. Esse procedimento é o mesmo utili- zado em diversos outros trabalhos na literatura (Oliveira and Vali, 1995; Frank et al., 2006). A concentração $c$ é então determinada dividindo-se esse número pelo volume de amostragem, ou seja: $c=n_{g} / v_{a}$.

\section{FERRAMENTAS DE PROCESSAMENTO DIGITAL DE IMAGENS UTILIZADAS NO CCNC-SDCC}

Como mencionado anteriormente o número de partículas ativadas na câmara de nuvens pode ser obtido de forma indireta, através da medição da intensidade de luz espalhada pela gotícula de água ao cruzar um feixe de luz, ou direta pela contagem das gotículas através das técnicas de visão computacional.

O processamento digital de imagens, para contagem automática do número de $\mathrm{CCN}$ ativados reportado por Frank et al, leva em consideração a quantidade de objetos brancos e de pixels brancos na imagem para calcular a quantidade de gotículas (Frank et al., 2006). Correções empíricas são utilizadas para os casos de concentrações mais elevadas, onde o efeito de sobreposições de gotículas é mais acentuado. Essa técnica apresentou resultados razoáveis para concentrações abaixo de $600 \mathrm{~cm}^{-3}$, mas para concentrações mais altas, encontradas em muitas situações de interesse, os resultados mostram uma subestimação sistemática do número de partículas ativadas (Frank et al., 2006; Rose et al., 2008).

A segmentação de objetos sobrepostos em imagens tem sido bastante estudada, uma vez que esse é um problema cuja solução robusta é fundamental em vários processos de automação (Schmitt and Reetz, 2009; Albuquerque et al., 2010; Pavlidis and Horowitz, 1974; Felix et al., 2009; Malpica et al., 1997). O método da transformada watershed tem sido utilizado em diversas aplicações onde o problema da segmentação de objetos sobrepostos está presente nas imagens (Yang et al., 2006; Mao et al., 2006; Parvati et al., 2008; Chen et al., 2009; Xie et al., 2009).

Nesse trabalho, propõe-se a aplicação da transformação $w a$ tershed associada à binarização por limiar e transformação de distância para contagem de gotículas originadas da ativação de CCN no CCNC construído. Essas transformações são descritas brevemente a seguir.

A transformação de distância, consiste em converter uma imagem digital binária em uma outra representada em níveis de cinza. Esta transformação tem como base a substituição de um pixel branco, por outro cujo valor representa a distância deste pixel branco a um pixel preto mais próximo conforme a equação dada por 


$$
\begin{aligned}
& d(x, y)=\min \operatorname{dist}\left\{(x, y),\left(x_{i}, y_{i}\right)\right\}, \\
& \left(x_{i}, y_{i}\right) \in \text { borda do objeto. }
\end{aligned}
$$

Algumas métricas para o cálculo da distância podem ser utilizadas como, por exemplo, a Euclidiana, City Block e Chessboard (Fabbri et al., 2008). Neste trabalho, devido à simplicidade computacional, utiliza-se a métrica Chessboard. $\mathrm{Na}$ Figura 3 é apresentada uma imagem resultante desta transformação aplicada a uma imagem binária.

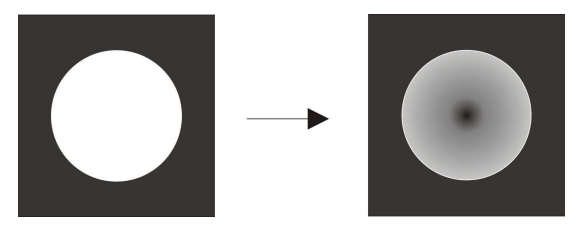

Figura 3: Exemplo de uma Transformação de Distância aplicada a uma imagem binária.

Um dos problemas mais complexos em visão computacional é a segmentação de imagens digitais. Segmentar uma imagem consiste em separar objetos presentes numa dada imagem. Existem várias técnicas de segmentação e duas técnicas são utilizadas neste trabalho: a binarização por limiar e a transformação que define a linha divisora de bacias hidrográficas ou simplesmente transformada wahershed (Digabel and Lantuéjoul, 1978; Lantuéjoul, 1978; Vincent and Soille, 1991; Beucher and Lantuéjoul, 1979).

A binarização por limiar consiste em gerar uma imagem binária $g(x, y)$, a partir de uma imagem em tons de cinza, $f(x, y)$, considerando um valor de limiar $k$ segundo a relação (Gonzalez and Woods, 2007)

$$
g(x, y)= \begin{cases}0, & \text { se } f(x, y)<k \\ 1, & \text { se } f(x, y) \geq k\end{cases}
$$

A transformada watershed consiste em considerar a imagem como sendo um relevo topográfico onde cada altura deste relevo é associada a uma intensidade de cinza. Ao se promover a inundação dos vales (bacias hidrográficas ou de captação), a partir de seus mínimos locais, e construindo-se diques, sempre que necessário para evitar-se transbordamento, destacam-se as linhas divisoras de bacias (watershed), produzindo desta forma a segmentação dos objetos presentes na imagem. Um eficiente algoritmo para realização da transformada watershed por imersão é proposto por Luc Vincent and Pierre Soille e é expressa na equação a seguir: (Vincent and Soille, 1991).

$$
\begin{gathered}
X_{h_{\min }}=T_{h_{\min }}(I), \\
X_{h+1}=\min _{h+1} \cup I Z_{T_{h+1}(I)}\left(X_{h}\right), \\
\forall h \in\left[h_{\min }, h_{\max }-1\right] .
\end{gathered}
$$

A ideia básica da transformada watershed é mostrada na Figura 4. Na subfigura (a) é apresentada uma superfície topográfica de um terreno onde são visíveis alguns vales (bacias hidrográficas). Na subfigura (b), com o processo de inundação em andamento a partir dos mínimos locais, a mesma superfície é apresentada, vista de cima, onde se pode observar 6 vales sendo que o de $\mathrm{n}^{o} 6$ é pequeno e de pouca profundidade. Nas subfiguras seguintes (c, d, e) a inundação continua sendo que são construídos diques, sempre que necessário, para impedir a formação de uma única superfície de água. A inundação prossegue, bem como a construção dos diques, até atingir o ponto mais alto do terreno. O resultado final deste procedimento é a segmentação (separação) das 6 bacias como é mostrado na subfigura (f).
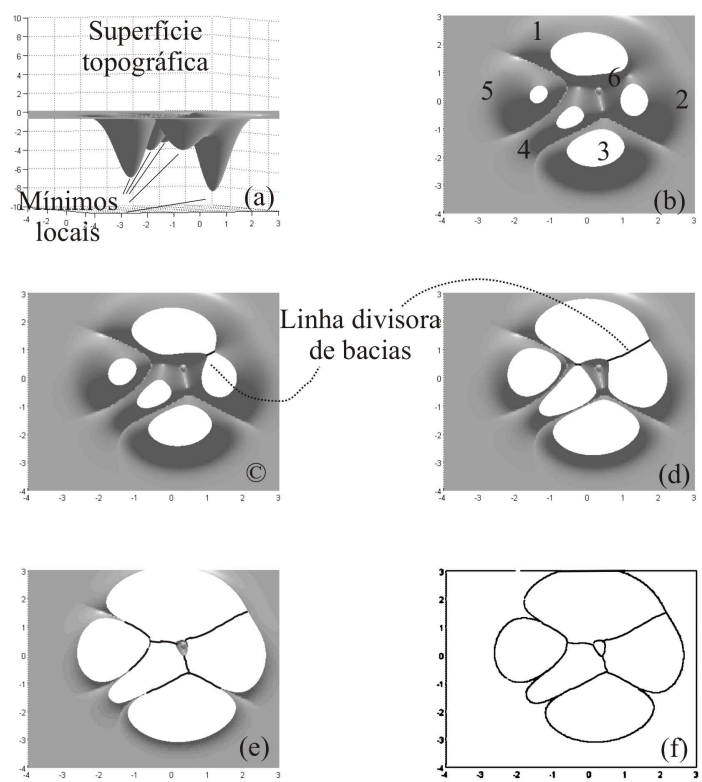

Figura 4: Ideia básica da transformada watershed, (a) superfície topográfica com alguns vales onde se destacam os mínimos locais; (b) vista superior da superfície com a inundação em andamento; (c); (d); (e) destaque para as linhas divisoras de água em construção; (f) objetos segmentados.

Um problema bastante comum, resultante da aplicação direta da transformada watershed, é a supersegmentação. Tal problema ocorre, pois as imagens, de um modo geral, possuem irregularidades locais ou ruído que normalmente são interpretados pela transformada watershed como sendo mínimos locais, ou seja, como bacias hidrográficas. Este é um problema amplamente abordado e encontra diversas so- 
luções na literatura (Roerdink and Meijster, 2001; Haris et al., 1998; Trémeau and Colantoni, 2000; Gauch, 1999; Meyer, 1994; Vincent and Soille, 1991). Neste trabalho, a supersegmentação é resolvida pela aplicação da transformação de distância como será mostrado mais adiante.

\section{METODOLOGIA}

O método empregado para a medição da concentração dos núcleos de condensação de nuvens neste CCNC-SDCC envolve dois aspectos importantes. O primeiro consiste na determinação do volume de amostragem a partir da definição da área da imagem a ser processada. O segundo consiste na aplicação das técnicas de processamento de imagens na detecção e contagem das gotículas de água formadas dentro do volume de amostragem. A avaliação do desempenho das técnicas utilizadas é feita submetendo-se, ao mesmo tempo, o CCNC-SDCC e um contador global de partículas a uma concentração de aerossóis produzida em condições controladas.

\subsection{Determinação do volume de amostra- gem da câmara de nuvens}

O valor do volume de amostragem, definido pela luz LASER que atravessa a câmara de nuvens, é um parâmetro crítico dentro do processo de medição de concentração dos $\mathrm{CCN}$. O volume de amostragem $V_{a}$, considerado cilíndrico, é definido por

$$
v_{a}=\pi \frac{d^{2}}{4} l,
$$

em que $d$ é o diâmetro da luz LASER e $l$ é o comprimento da luz LASER na região de interesse. Neste caso, $v_{a}$ é todo o volume iluminado pela luz LASER dentro da câmara de nuvens.

Este volume de amostragem encontra-se aproximadamente a meia altura da câmara de nuvens, pois, conforme é mostrado na Figura 2(c) é nesta região onde o valor da supersaturação é máximo.

Os parâmetros $d$ e $l$ são a altura e o comprimento da área retangular na imagem onde as gotículas estão iluminadas (volume de amostragem considerado cilíndrico visto de frente). O procedimento para determinar estes parâmetros consiste localizar as coordenadas em pixels dos vértices do retângulo e correlacioná-las a um valor de comprimento. Para tal, os seguintes passos são realizados: 1- registra-se a imagem de um papel milimetrado colocado no centro da câmara de nuvens e alinhado com a luz LASER de modo a permitir a calibração da relação pixels/milímetros; 2 - registra-se um número suficiente de imagens, com a presença de gotículas, de maneira tal que a sobreposição das imagens possa definir completamente a região iluminada pela luz LASER indicando as coordenadas do retângulo da imagem a ser processada. A partir da relação pixels/milímetros e das coordenadas do retângulo obtém-se $d$ e $l$.

Três imagens do processo de calibração estão apresentadas na Figura 5: (a) papel milimetrado posicionado no centro da câmara de nuvens, exatamente por onde a luz LASER passa; (b) gotículas formadas dentro da câmara de nuvens; (c) sobreposição de imagens, todas com gotículas, revelando totalmente o diâmetro $d$ da luz LASER. Feito isto, é possível construir um gráfico de distribuição de quantidade de pixels de valor 1 (branco) ao longo da altura de toda a imagem $i(y)$ e se obter o diâmetro $d$, dada por

$$
i(y)=\sum_{x=0}^{m-1} g(x, y), y=0, \ldots, n-1,
$$

em que $m$ e $n$ são o número de pixels da imagem no eixo horizontal e vertical, respectivamente.

Quanto ao comprimento $l$, este deve ser considerado como sendo praticamente todo o comprimento da imagem, evitando-se apenas uma pequena região próxima das bordas laterais de modo a prevenir erros no processamento.

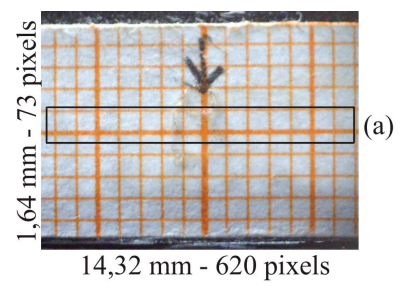

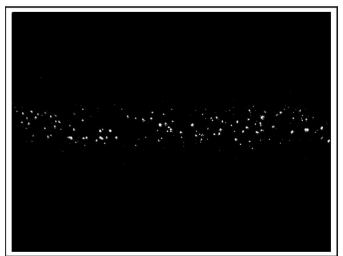

(b)

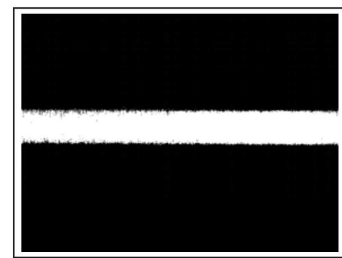

(c)
Figura 5: Imagens do processo de calibração, (a) imagem de papel milimetrado no centro da câmara de nuvens; (b) imagem das gotículas dentro da câmara de nuvens; (c) resultado de 2000 imagens sobrepostas.

\subsection{Processamento das imagens}

O processamento das imagens consiste na aplicação das técnicas anteriormente descritas e é apresentado no diagrama de blocos da Figura 6, exibindo o fluxo de análise da imagem de entrada correspondendo às saídas parciais. 


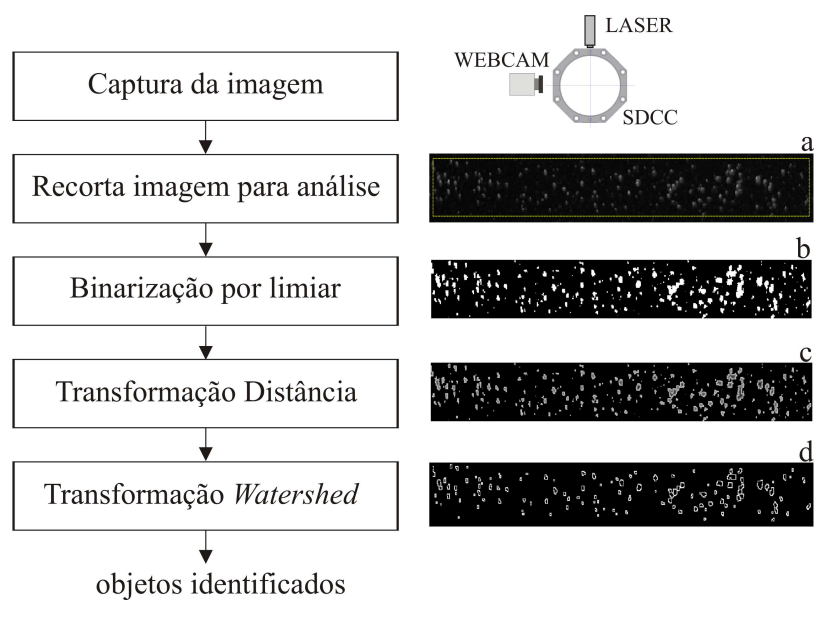

Figura 6: Diagrama de blocos do processamento das imagens e as saídas correspondentes a cada processo.

Para captura das imagens utiliza-se uma câmera de vídeo com uma resolução de $640 \times 480$ pixels instalada na janela de fotografia da câmara de nuvens. Em seguida, um recorte retangular desta imagem inicial é realizado. As coordenadas do retângulo são definidas no processo de determinação do volume de amostragem. A imagem assim produzida contém apenas a informação a ser processada, sendo, pois, usada como a entrada efetiva de dados.

O segundo passo consiste em aplicar uma binarização por limiar com o objetivo de realizar uma primeira segmentação da imagem segundo a equação 2. Assim, um nível de limiar de cinza mínimo (intensidade de luz) deve ser definido de modo que garanta que a informação é oriunda de dentro do volume de amostragem e é efetivamente uma gotícula de água. A definição deste limiar é feita medindo-se o valor médio das intensidades dos níveis de cinza dos pixels sem formação de gotículas dentro da câmara de nuvens. Neste trabalho, o valor médio encontrado é de 25. Desta forma, os pixels cujos valores de intensidade de nível de cinza variam de 0 a 25 são substituídos por zero (preto) e acima deste valor são admitidos iguais a 1 (branco). Assim, obtém-se uma imagem binarizada conforme está mostrado na parte b da Figura 6, produzida por este processo.

A etapa seguinte consiste na construção de mínimos locais artificiais, utilizando-se a transformação de distância conforme a equação 1 . Isto tem como objetivo produzir um mínimo local em cada gotícula de água. Considerando-se que na imagem binarizada as gotículas possuem um formato cujos os pontos do contorno encontram-se aproximadamente equidistantes do centro da mesma, a transformação de distância produz, neste caso, garantidamente um único mínimo local em cada gotícula. No caso de gotículas parcialmente sobrepostas mais de um mínimo local é produzido. A sobreposição de gotículas dentro da câmara de nuvens é comum acontecer em altas concentrações. O resultado deste processamento é mostrado na parte c da Figura 6.

A partir da construção dos mínimos locais, a transformada watershed é aplicada, conforme a equação 3 , ao resultado mostrado na parte c da Figura 6 conforme o algoritmo proposto por Luc Vincent and Pierre Soille (Vincent and Soille, 1991). O objetivo principal da aplicação desta transformada é identificar gotículas de água parcialmente sobrepostas. Como no processo de construção dos mínimos locais foram construídos apenas um mínimo local por gotícula, fica garantido a não ocorrência do fenômeno da supersegmentação. O Resultado deste processamento é mostrado na parte $\mathrm{d}$ da Figura 6.

\subsection{Bancada geradora de aerossóis}

A metodologia para avaliação de desempenho do CCNCSDCC consiste na mesma que é utilizada em diversos trabalhos na área (Frank et al., 2006; Rose et al., 2008; Zhang et al., 2008). Isto é, foi realizada uma comparação entre um contador de partículas condensáveis (Ultrafine Condensation Particle Counter Model 3025) e o CCNC-SDCC. Para tal, foi utilizada, no Laboratório de Química da Partícula do Instituto Max Planck na Universidade de Mainz - Alemanha, uma bancada capaz de produzir aerossóis sob condições controladas de concentração, bem como de diâmetro de partícula. Na Figura 7 são apresentados os diversos componentes da bancada: compressor, armadilha, atomizador, reservatório com uma solução de sulfato de amônia, secador, classificador eletrostático, e o contador de partículas.

O processo de geração de aerossóis inicia-se em um compressor com saída de ar filtrado. Em seguida, este ar filtrado passa por uma armadilha de água. Neste ponto, o ar pressurizado, filtrado e seco é injetado no atomizador TSI3076 que esta acoplado a um reservatório, contendo uma solução de sulfato de amônia $\left(\mathrm{NH}_{4}\right)_{2} \mathrm{SO}_{4}$. Outras substâncias higroscópicas podem ser utilizadas como, por exemplo, nitrato de amônia $\mathrm{NH}_{4} \mathrm{NO}_{3}$, cloreto de sódio $(\mathrm{NaCl})$ e etc. $\mathrm{Na}$ saída deste atomizador, tem-se os $\mathrm{CCN}$ propriamente dito, embora polidisperssivo e com uma umidade muito alta. A remoção da umidade é feita por um secador a base de silicagel.

Como o processo de condensação do vapor de água é altamente dependente do tamanho do aerossol, ou seja, do tamanho do núcleo de condensação, exige-se um controle deste parâmetro. Isto é, exige-se que o aerossol seja monodispersivo. Para tal, utiliza-se na saída do secador um classificador eletrostático, que no caso é o TSI3080. Por fim, a medição da concentração das partículas é feita por um contador de partículas condensáveis (CPC), no caso o TSI3025A. 


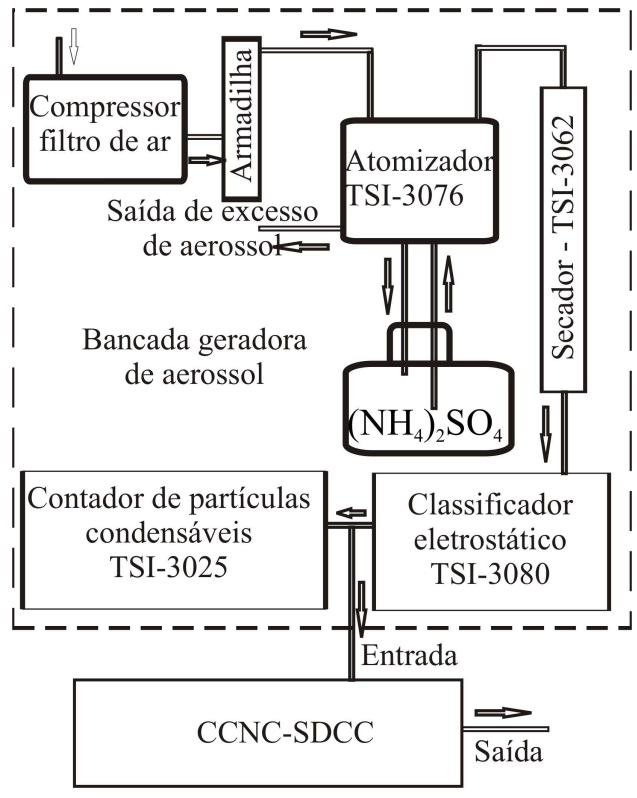

Figura 7: Bancada geradora de aerossóis.

O contador de partículas condensáveis TSI3025A é capaz de medir aerossóis com um diâmetro de $3 \mathrm{~nm}$ até $3 \mu \mathrm{m}$ numa faixa de concentração de 0 até $9,99 \cdot 10^{4}$ partículas $\cdot \mathrm{cm}^{-3}$. Em condições normais, seu sensor de partículas opera em uma atmosfera saturada de Butanol-1. Esta característica o torna sensível, não apenas aos aerossóis responsáveis pela formação das nuvens e das chuvas (CCN), mas a qualquer aerossol. Por esta razão, no processo de comparação, normalmente se utiliza apenas aerossóis higroscópicos (como, por exemplo, o sulfato de amônia, nitrato de amônia ou cloreto de sódio) que são capazes de sensibilizar tanto o CCNCSDCC quanto o CPC TSI3025A.

\subsection{Aspectos práticos do processo de comparação}

Considerando-se que este trabalho envolve fenômenos físicos reais, algumas considerações do ponto de vista prático, no que diz respeito ao ajuste da concentração sua variação e seus transitórios, são importantes de serem postas.

\subsubsection{Ajuste da concentração}

O ajuste da concentração é feito de forma empírica. Isto é, dissolve-se o sulfato de amônia em água destilada em uma proporção que corresponda a uma dada concentração que se deseja medir no CPC. Pequenas correções podem ser realizadas, adicionando-se água destilada ou sulfato de amônia para redução ou aumento da concentração, respectivamente. Outra forma de se obter pequenos ajustes é aumentando ou diminuindo a pressão de ar comprimido no sistema através de uma válvula.

Foram definidas três faixas de concentrações para realização dos experimentos de comparação:

- baixa $\ldots c<1500$ partículas $\cdot \mathrm{cm}^{-3}$

- média......1500 $<c<3000$ partículas $\cdot \mathrm{cm}^{-3}$

- alta...........c $>3000$ partículas $\cdot \mathrm{cm}^{-3}$

Estas faixas de valores de concentração de partículas foram escolhidas, pelo fato destas representarem situações normalmente encontradas na natureza (Andreae et al., 2004).

\subsubsection{Variação da concentração}

A concentração medida pelo CPC varia ao logo do tempo como é mostrado na Figura 8. Esta variação é devida a uma remoção da água e do sulfato em proporções diferentes durante o processo de produção de aerossol. Este fenômeno tem forte implicação no procedimento de comparação. Isto porque as médias de medições obtidas em um longo período de tempo não fazem sentido neste caso, mas sim a comparação da medida obtida pelo CCNC-SDCC e a medida obtida pelo CPC em um curto espaço de tempo.

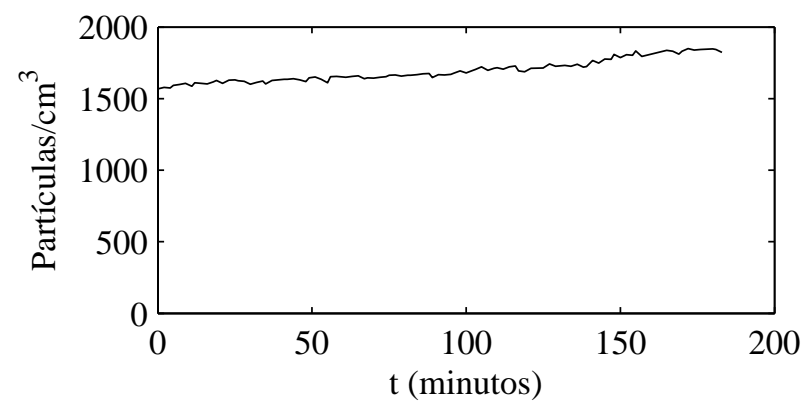

Figura 8: Variação da concentração da bancada geradora de aerossóis.

\subsubsection{Transitórios}

O CPC foi projetado para trabalhar com um fluxo contínuo de aerossol. O modelo TSI3025A, utilizado como referência neste trabalho, possui uma bomba que procura sempre manter o fluxo de 0,3 litros/min de aerossol constante em seu sistema de medição. Como o CCNC-SDCC encontra-se em paralelo com o CPC, ao ser acionada a sua bomba para a realização da medição, este sofre uma alteração momentânea de fluxo, como mostra as grandes variações destacadas na região (b) da Figura 9. Estes transitórios devem ser descartados no processo de comparação. 


\subsubsection{Procedimentos adotados para comparação}

Os efeitos da variação da concentração ao longo do tempo, bem como os efeitos transitórios impostos ao CPC devido ao princípio operacional do CCNC-SDCC, impõem restrições nos dados de saída do CPC. Assim, consideram-se como dados válidos do CPC, para efeito de comparação, as 50 últimas medições antes do acionamento da bomba do CCNC-SDCC. Na Figura 9 são destacadas a região de interesse (a) e a descartada (b) do sinal do CPC no processo de comparação.

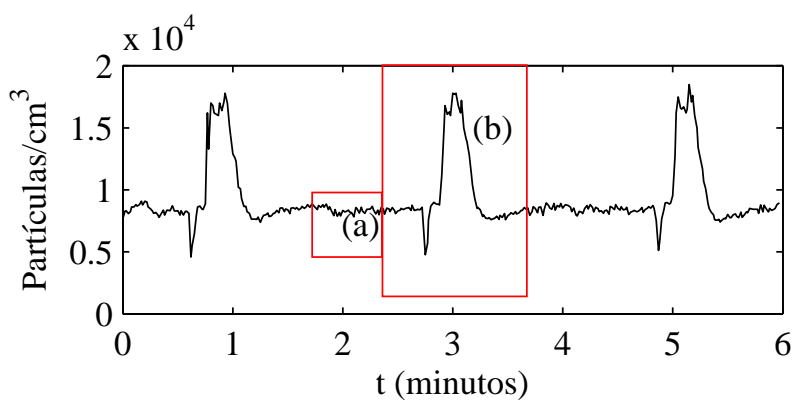

Figura 9: Concentração medida pelo CPC com destaque para dois tipos de regiões.

\section{RESULTADOS E DISCUSSÃO}

As coordenadas da região retangular da imagem com gotículas iluminadas, área efetiva de dados, foram obtidas a partir da utilização de 2000 imagens sobrepostas conforme é mostrado na Figura 5(c). Consideram-se como coordenadas da altura do retângulo as posições inicial e final que contêm pelo menos $1 \%$ do número máximo de ocorrências de pixels com valor 1. Na Figura 10 é apresentado um gráfico, conforme a equação 5 , com o número de vezes em que uma determinada posição da altura teve seu pixel com valor 1, independente da posição horizontal. Já no caso das coordenadas que definem o comprimento do retângulo, estas foram escolhidas de modo a se obter a maior área possível para análise. Entretanto, desprezou-se 10 pixels nas laterais, de modo a garantir uma região segura de processamento de dados. Deste modo, os valores das coordenadas para os vértices superior esquerdo e inferior direito são respectivamente $(10,193)$ e $(630,266)$, os quais definem os valores da altura e do comprimento da área iluminada, respectivamente de 73 e 620 pixels.

A partir da relação pixels/milímetros (43,29 pixels/milímetro na horizontal e 44,50 pixels/milímetro na vertical), obtida através da análise da imagem mostrada na Figura 5a, e da altura e do comprimento em pixels definidos anteriormente, obtém-se o valor do volume de amostragem conforme a equação $4 \mathrm{Va}=30,27 \pm 1,76 \mathrm{~mm}^{3}$. O valor da incerteza do volume $\left(1,76 \mathrm{~mm}^{3}\right)$ foi calculado considerando-se um pos-

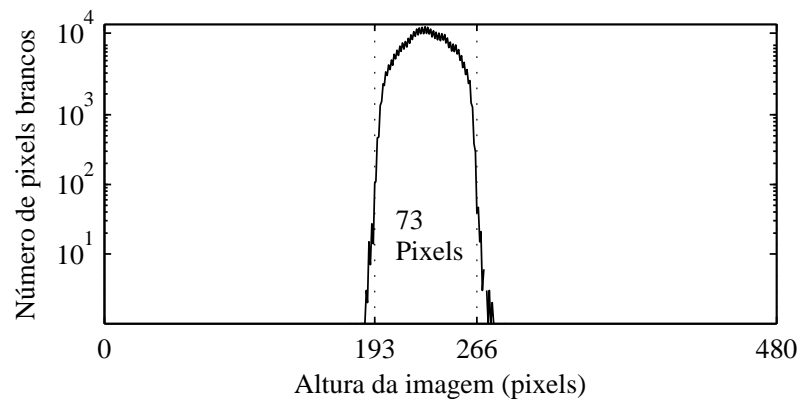

Figura 10: Gráfico de distribuição de pixels brancos (valor 1) ao longo da altura da imagem conforme a equação 5 .

sível erro de $\pm 1,0 \mathrm{~mm}$ no posicionamento do papel milimetrado em relação ao feixe de luz LASER.

No que diz respeito à avaliação do desempenho do CCNCSDCC, o processo de comparação apresentado na forma das Figuras 11 e 12 indica uma forte correlação entre o CPC e o CCNC-SDCC, no caso o coeficiente de correlação foi de 0,99. A faixa de concentração analisada vai de 500 partículas $/ \mathrm{cm}^{3}$ até 4500 partículas $/ \mathrm{cm}^{3}$. Esta é uma faixa bastante ampla e abrange desde condições comumente encontradas nos processos de formação de nuvens no oceano ou floresta (em situações de baixa poluição) até de formação de nuvens produzidas por plumas de fumaça oriundas de queima de florestas (Andreae et al., 2004).

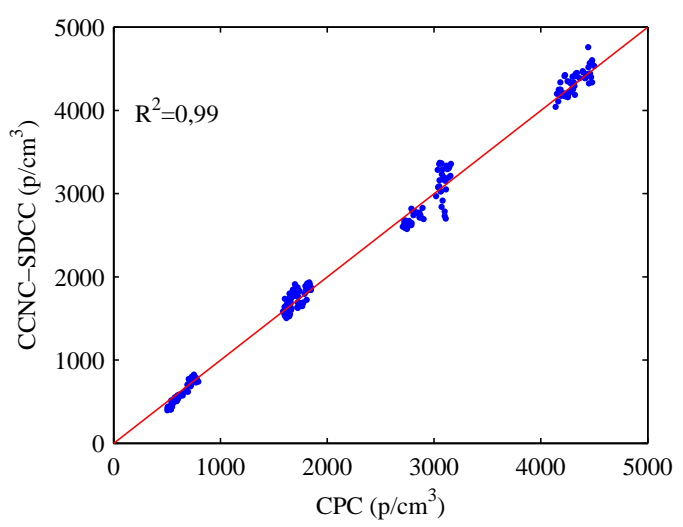

Figura 11: Correlação entre CPC e CCNC-SDCC utilizando aerossóis monodisperssivo de sulfato de amônia com diâmetro de $300 \mathrm{~nm}$.

É importante ressaltar que a única calibração feita no CCNCSDCC é aquela realizada na determinação do volume de amostragem. Isto é, não é necessário nenhum ajuste nos dados obtidos mesmo em situações de alta concentração onde o fenômeno da sobreposição de gotículas nas imagens é co- 


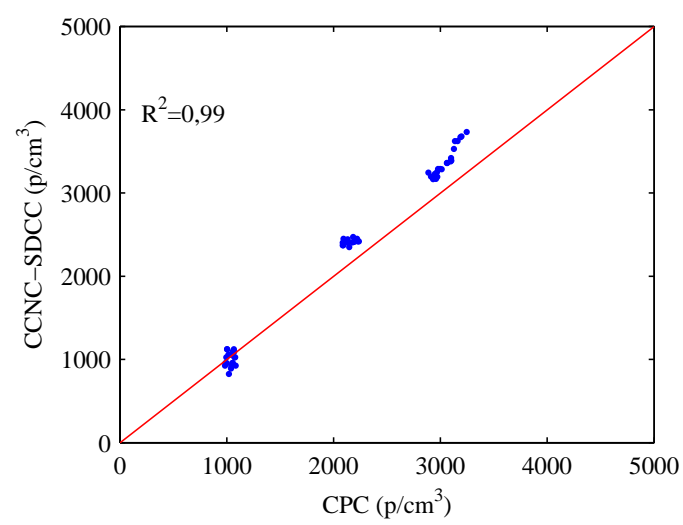

Figura 12: Correlação entre CPC e CCNC-SDCC utilizando aerossol monodisperssivo de sulfato de amônia com diâmetro de $100 \mathrm{~nm}$.

mum de ocorrer. Este resultado demonstra claramente que o procedimento adotado para a medição do volume de amostragem, bem como, as técnicas adotadas para contagem das gotículas são adequadas para esta aplicação. Desta forma, este CCNC-SDCC dispensa a utilização de um sofisticado sistema de produção de aerossóis para calibração.

Outro aspecto importante que deve ser ressaltado é o que diz respeito à dispersão dos dados. Na Figura 13 observa-se que o CCNC-SDCC possui um desvio padrão relativo de $12 \%$ enquanto que o do CPC é de 7,4\% para o mesmo conjunto de dados. Entretanto, na média os valores são muito próximos, conforme é demonstrado através das linhas de tendências desenhadas na Figura 13.

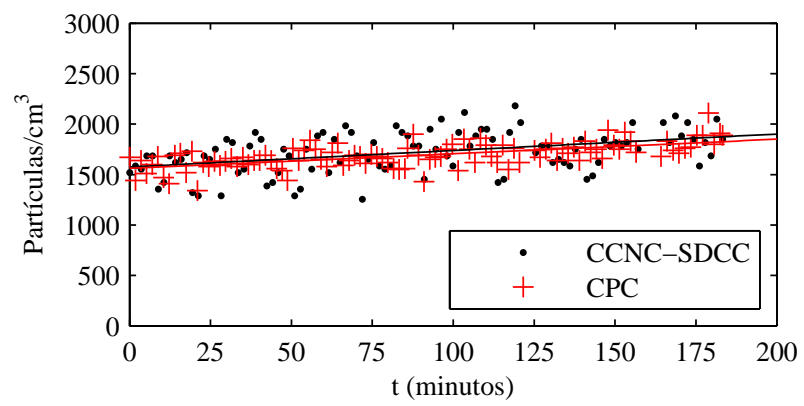

Figura 13: Comparação da dispersão dos dados entre CPC e o CCNC-SDCC.

Quanto ao risco de supersegmentação, os procedimentos adotados mostraram-se eficientes no caso da segmentação das gotículas de água considerando-se que 40.000 imagens foram processadas e nenhum único caso de supersegmentação foi constatado.
Com relação às incertezas no processo de medição alguns aspectos devem ser ressaltados. O primeiro refere-se à exatidão do valor da diferença de temperatura entre as placas da câmara de nuvens. Os sensores de temperatura utilizados foram testados sob as mesmas condições e a diferença de temperatura nunca foi maior do que $0,0625^{\circ} \mathrm{C}$. Isto garante um erro menor do que $3,0 \%$ no valor da supersaturação, de acordo com simulações realizadas a partir das equações que são descritas na literatura (Frank et al., 2006). O segundo aspecto diz respeito ao pequeno volume de amostragem produzido pela luz LASER $\left(V_{a}=30,27 \pm 1,76 \mathrm{~mm}^{3}\right)$. Como não se pode garantir uma distribuição uniforme dos $\mathrm{CCN}$ dentro da câmara de nuvens, algumas medições devem ser realizadas para se obter um valor representativo da concentração dos CCN. Por fim, o terceiro aspecto diz respeito à exatidão do CPC (TSI 3025A), assumido como referência. Conforme especificações do fabricante este CPC possui uma acurácia de $10 \%$ até $10^{4} / \mathrm{cm}^{3}$ e considerando-se que a correlação entre os instrumentos é praticamente 1.0 podemos assumir que este CCNC-SDCC tem a mesma classe de acurácia.

\section{CONCLUSÕES E CONTRIBUIÇÕES}

Este artigo propõe um sistema para a medição automática da concentração de núcleos de condensação de nuvens utilizando técnicas de processamento digital de imagens. Os resultados obtidos e apresentados mostram que o sistema proposto é eficiente na determinação da concentração dos núcleos de condensação de nuvens a partir do CCNC-SDCC.

$\mathrm{O}$ algoritmo de processamento de imagem utilizado (binarização, transformada de distância e transformada watershed) tornou a câmara de nuvens estática por difusão significativamente eficiente para medir concentrações de $\mathrm{CCN}$ acima da capacidade dos equipamentos similares. Este desempenho é resultado direto da aptidão natural do algoritmo de identificar, nas imagens analisadas, gotas parcialmente sobrepostas.

Outra contribuição igualmente importante consiste na metodologia apresentada para determinação do volume de amostragem da câmara de nuvens, permitindo a construção de um CCNC-SDCC sem a necessidade de bancadas de calibração ou de equipamentos de referência. Por fim, também como contribuição podem-se considerar a redução de peso, dimensões e consumo de energia por este equipamento.

\section{AGRADECIMENTOS}

Ao Laboratório de Química da Partícula do Instituto MaxPlanck, ao Laboratório de Teleinformática da Universidade Federal do Ceará, ao Curso de Física da Universidade Estadual do Ceará, a Fundação Cearense de Meteorologia e Recursos Hídricos e, por fim, a Fundação Cearense de Apoio 
ao Desenvolvimento Científico pelos suportes técnico e financeiro.

\section{REFERÊNCIAS}

Albuquerque, V. H. C. D., Tavares, J. M. R. and Cortez, P. C. (2010). Quantification of the microstructures of hypoeutectic white cast iron using mathematical morphology and an artificial neural network., International Journal of Microstructure and Materials Properties 5: 52-64.

Andreae, M. O., D.Rosenfeld, Artaxo, P., Costa, A. A., Longo, G. F. K. and F.Silva-Dias, M. A. (2004). Smoking rain clouds over the amazon, Science 303: 13371342.

Beucher, S. and Lantuéjoul, C. (1979). Use of watersheds in contour detection, International Workshop on Image Processing, Real-Time Edge and Motion Detection/Estimation, Rennes.

Chen, L., Jiang, M. and Chen, J. (2009). Image segmentation using iterative watersheding plus ridge detection, IEEE International Conference on Image Processing.

Digabel, H. and Lantuéjoul, C. (1978). Iterative algorithms, in Caen (ed.), Actes du Second Symposium Europt'een dŠAnalyse Quantitative des Microstructuresen Sciences des Matt'eriaux, Biologie et Mt'edecine, , pp. 85-99.

Fabbri, R., da F. Costa, L., Torelli, J. C. and M.Bruno, O. (2008). 2d euclidean distance transform algorithms: A comparative survey, ACM Computing Surveys 40(1): 144.

Felix, J., Cortez, P. C., R.C.S, C., Fortaleza, S. C. B., PEREIRA, E. D. B. and HOLANDA, M. A. (2009). Avaliação computacional de enfisema pulmonar em tc: comparação entre um sistema desenvolvido localmente e um sistema de uso livre, Jornal Brasileiro de Pneumologia 35: 868-876.

Frank, G. P., Dusek, U. and Andreae, M. O. (2006). Technical note: Characterization of a static thermalgradient ccn counter, Atmospheric Chemistry and Physics 6: 2151-2174.

Gauch, J. M. (1999). Image segmentation and analysis via multiscale gradient watershed hierarchies, IEEE Transactions on Image Processing 8: 69-79.

Gonzalez, R. C. and Woods, R. E. (2007). Digital Image Processing, 3 edn, -.

Haris, K., Efstratiadis, S. N., Maglaveras, N. and Katsaggelos, A. K. (1998). Hybrid image segmentation using watersheds and fast region merging, IEEE TRANSACTIONS ON IMAGE PROCESSING 7: 1684-1699.

Khain, A. P. (2009). Notes on state-of-the-art investigations of aerosol effects on precipitation: a critical review, Environmental Research Letters 4: 015004.

Lantuéjoul, C. (1978). La squelettisation et son application aux mesures topologiques des mosa ques polycristallines, $\mathrm{PhD}$ thesis, Ecole des Mines, Paris.

Loarie, S. R., Duffy, P. B., Hamilton, H., Asner, G. P., Field, C. B. and Ackerly, D. D. (2009). The velocity of climate change, Nature 462: 1052-1055.

Malpica, N., de Solórzano, C. O., Vaquero, J. J., Santos, A., Vallcorba, I., García-Sagredo, J. M. and del Pozo, F. (1997). Applying watershed algorithms to the segmentation of clustered nuclei, Cytometry 28: 289-297.

Mao, K. Z., Zhao, P. and Tan, P.-H. (2006). Supervised learning-based cell image segmentation for p53 immunohistochemistry, IEEE Transactions on Biomedical Engineering 53: 1153-1163.

McMurry, P. H. (2000). A review of atmospheric aerosol measurements, Atmospheric Environment 34: 1959-1999.

Meyer, F. (1994). Topographic distance and watershed lines, Signal Processing 38: 113-125.

Nenes, A., Chuang, P. Y., Flagan, R. C. and Seinfeld, J. H. (2001). A theoretical analysis of cloud condensation nucleus (ccn) instruments, Journal of Geophysical Research 106: 3449-3474.

Oliveira, J. and Vali, G. (1995). Calibration of a photoelectric cloud condensation, Atmospheric Research 38: 237248.

Parvati, K., Rao, B. S. P. and Das, M. M. (2008). Image segmentation using gray-scale morphology and markercontrolled watershed transformation, Discrete Dynamics in Nature and Society pp. 1-8.

Pavlidis, T. and Horowitz, S. (1974). Segmentation of plane curves, IEEE Transactions on Computers C-23: 860 870 .

Pinheiro, F. G. M. (1999). Cancelador adaptativo de ruído para um contador de núcleos de condensaçãode nuvens, Master's thesis, Universidade Federal do Ceará.

Roberts, G. C., Andreae, M. O., Zhou, J. and Artaxo, P. (2001). Cloud condensation nuclei in the amazon basis: Marine conditions over continent?, Geophysical Reasearch Letters 28(14): 2807-2810. 
Roerdink, J. B. T. M. and Meijster, A. (2001). The watershed transform: Definitions, algorithms and parallelization strategies, Fundamenta Informaticae 41: 187-228.

Rose, D., Gunthe, S. S., Mikhailov, E., Frank, G. P., Dusek1, U., Andreae1, M. O. and Pöschl, U. (2008). Calibration and measurement uncertainties of a continuous-flow cloud condensation nuclei counter (dmt-cenc): Ccn activation of ammonium sulfate and sodium chloride aerosol particles in theory and experiment, Atmospheric Chemistry and Physics 8: 1153-1179.

Rosenfeld, D. (2006). Aerosols, clouds, and climate, Science

Schmitt, O. and Reetz, S. (2009). On the decomposition of cell clusters, Math Imaging Vis 33: 85-103.

Trémeau, A. and Colantoni, P. (2000). Regions adjacency graph applied to color image segmentation, IEEE Transactions on Image Processing 9: 735-744.

Twomey, S. (1963). Measurements of natural cloud nuclei, Journal of Atmospheric Research 1: 101-105.

Verdes, P. F. (2007). Global warming is driven by anthropogenic emissions: Atime series analysis approach, Physical Review Letters 99.

Vianello, R. L. and Alves, A. R. (1991). Meteorologia Básica e Aplicações, Universidade Federal de Viçosa.

Vincent, L. and Soille, P. (1991). Watersheds in digital spaces: An efficient algorithm based on immersion simulations, IEE Transactions on Pattern Analysis and Machine Intelligence 13: 583-598.

Wagner, W. and PruSS, A. (2002). The iapws formulation 1995 for the thermodynamic properties of ordinary water substance for general and scientific use, Journal of Physical and Chemical Reference Data 31: 387-535.

Working Group I of the Intergovernmental Panel on Climate Change (2007). Climate change 2007: The physical science basis, Technical report, World Meteorological Organization and United Nations Environment Programme.

Xie, Z. X., Yu, D. R., Zhang, J., Yang, S. H., and Hu, Q. H. (2009). Properties of magnetic elements in the quiet sun using the marker-controlled watershed method, Astronomy \& Astrophysics 505: 801-810.

Yang, X., Li, H., and Zhou, X. (2006). Nuclei segmentation using marker-controlled watershed, tracking using mean-shift, and kalman filter in time-lapse microscopy, IEEE Transactions on Circuits and Systems 53: 24052414.
Zhang, D., Moore, K. F., Friedl, R. R. and Leu, M.-T. (2008). Design and characterization of a horizontal thermal gradient cloud condensation nucleus spectrometer, Journal of Aerosol Science 39: 30-39. 